\title{
1 Evidence of reduced individual heterogeneity in adult survival of
}

\section{2 long-lived species}

3 Guillaume Péron ${ }^{1,2}$, Jean-Michel Gaillard ${ }^{2}$, Christophe Barbraud ${ }^{3}$, Christophe Bonenfant $^{2}$, 4 Anne Charmantier ${ }^{4}$, Rémi Choquet ${ }^{4}$, Tim Coulson ${ }^{5}$, Vladimir Grosbois ${ }^{6}$, Anne Loison ${ }^{7,8}$, 5 Gilbert Marzolin ${ }^{4}$, Norman Owen-Smith ${ }^{9}$, Déborah Pardo $^{3}$, Floriane Plard ${ }^{2,10}$, Roger Pradel ${ }^{4}$, 6 Carole Toïgo ${ }^{11}$, Olivier Gimenez ${ }^{4}$

71 Smithsonian Conservation Biology Institute, National Zoological Park, Front Royal, 8 Virginia 22630, USA

92 UMR CNRS 5558 - LBBE "Biométrie et Biologie Évolutive", UCB Lyon 1 - Bât. Grégor

10 Mendel, 43 bd du 11 novembre 1918, 69622 Villeurbanne cedex, France

113 Centre d'Etudes Biologiques de Chizé UMR 7372 CNRS / Université La Rochelle,

1279360 Villiers en Bois, France

134 CEFE UMR 5175, CNRS - Université de Montpellier - Université Paul-Valéry

14 Montpellier - EPHE, 1919 Route de Mende, 34293 Montpellier cedex 5, France

155 Department of Zoology, University of Oxford, OX1 3PS, UK

166 UR AGIRs - Animal et Gestion Intégrée des Risques, TA C 22/E Campus International

17 Baillarguet, 34398 Montpellier cedex 5, France

187 Laboratoire d'Ecologie Alpine, Université de Savoie Mont-Blanc, 73376 Le Bourget du

19 Lac, France

208 Laboratoire d'Ecologie Alpine, CNRS, 38000 Grenoble, France

219 Centre for African Ecology, School of Animal, Plant and Environmental Sciences,

22 University of the Witwatersrand, Wits 2050, South Africa

2310 Swiss Ornithological Institute, CH-6204 Sempach, Switzerland

2411 ONCFS - Unité Faune de Montagne, 5 allée de Bethléem, Z.I. de Mayencin, 38610

25 Gières, France 
28 The canalization hypothesis postulates that the rate at which trait variation generates variation

29 in the average individual fitness in a population determines how buffered traits are against

30 environmental and genetic factors. The ranking of a species on the slow-fast continuum - the

31 covariation among life-history traits describing species-specific life cycles along a gradient

32 going from a long life, slow maturity, and low annual reproductive output, to a short life, fast

33 maturity, and high annual reproductive output - strongly correlates with the relative fitness

34 impact of a given amount of variation in adult survival. Under the canalization hypothesis,

35 long-lived species are thus expected to display less individual heterogeneity in survival at the

36 onset of adulthood, when reproductive values peak, than short-lived species. We tested this

37 life history prediction by analysing long-term time series of individual-based data in nine

38 species of birds and mammals using capture-recapture models. We found that individual

39 heterogeneity in survival was higher in species with short generation time $(<3$ years $)$ than in

40 species with long generation time ( $>4$ years). Our findings provide the first piece of empirical

41 evidence for the canalization hypothesis at the individual level from the wild.

\section{KEYWORDS}

43 capture-recapture; comparative analyses; individual differences; life history evolution;

44 mixture models; random-effect models; vertebrates. 
45 Life history traits such as lifespan and reproductive rates are well known to co-vary, forming

46 life history strategies (Stearns 1976). In particular, a recurring pattern in cross-species

47 comparative demography is the existence of a slow-fast continuum of life histories going from

48 long-lived, late-maturing and slow-reproducing species to short-lived, early-maturing and

49 highly fecund species (see Gaillard et al. 2016 for a recent review). The continuum is in part

50 linked to variation in body mass, temperature, and development time (Harvey and Zammuto

51 1985; Gillooly et al. 2001) but still occurs when allometric relationships linking life history

52 traits and body mass or size have been accounted for (Stearns 1983; Brown and West 2000;

53 Gaillard et al. 2016), leading to the idea that the slow-fast continuum of life histories reflects

54 constraints or opportunities afforded by particular lifestyles (Brown and Sibly 2006), in

55 relation to or independently of energy allocation trade-offs (Kirkwood and Holliday 1979).

56 Irrespective of the mechanism(s) underlying this slow-fast continuum of life histories, the

57 ranking of a species along the continuum is known to correlate with the rate at which given

58 amounts of variation in life history traits generates variation in population growth rate (Pfister

59 1998). In species close to the slow end of the continuum, called long-lived species in the

60 following, variation in adult survival gives rise to the most variation in population growth rate

61 (Caswell 2001). As population growth rate represents the average fitness of the population

62 (Fisher 1930), individuals of long-lived species are therefore expected to display risk

63 spreading and risk avoidance tactics, both part of a bet-hedging strategy aimed at maximizing

64 survival probability (Gaillard and Yoccoz 2003; Koons et al. 2009; Nevoux et al. 2010).

65 These are in turn expected to buffer phenotypes against perturbations caused by genetic

66 (Stearns and Kawecki 1994) or environmental (Gaillard and Yoccoz 2003) factors. Such a

67 buffer effect is usually called a canalization process (sensu Waddington 1953). We therefore

68 predict adults in populations of long-lived species to have more similar survival probabilities

69 than adults in populations of short-lived species. A few previous studies have focused on the 
70 magnitude of temporal variation in demographic rates in relation to their demographic impact

71 (following Pfister's (1998) pioneer analysis). However, we are not aware of any study linking

72 the demographic impact of traits to between-individual variance, except studies of Drosophila

73 melanogaster in the lab (Stearns and Kawecki 1994). We took advantage of available long-

74 term time series of demographic data in the wild and of modern statistical methods to test for

75 the canalization of adult survival at the individual level in the wild. Under the canalization

76 hypothesis, we expected between-individual variance in adult survival to decrease from short-

77 to long-lived species.

\section{Material and methods}

\section{DATA SETS}

80 We studied nine species including four mammalian large herbivores - roe deer (Capreolus

81 capreolus; two populations), chamois (Rupicapra rupicapra), Alpine ibex (Capra ibex), and

82 greater kudu (Tragelaphus strepsiceros; two populations) - and five birds - black-headed gull

83 (Chroicocephalus ridibundus), blue tit (Cyanistes caeruleus), white-throated dipper (Cinclus

84 cinclus), snow petrel (Pagodroma nivea) and black-browed albatross (Thalassarche

85 melanophris). All were subjected to detailed long-term monitoring at the individual level

86 (Table S1 in supplementary material A). Individuals were uniquely marked at first capture and

87 physically recaptured or resighted later in life. Imperfect detection was accommodated using

88 capture-recapture (CR) models (Lebreton et al. 1992).

\section{INDIVIDUAL VARIATION IN SURVIVAL PROBABILITY}

90 We aim at comparing, across species, the within-species, between-individual variance in adult

91 survival. To do that we use the concept of frailty (sensu Vaupel et al. 1979). Frailty

92 corresponds to the mortality risk of a given individual at a given age relative to the population

93 average. In this study we measure frailty via the variation among individuals in the intercept 
94 of the age-survival curve, i.e., the variance in the survival probability at the onset of adulthood

95 (the age at maturity when reproductive values peak). In other words, a frailty value is assigned

96 to each individual at the onset of adulthood and is conserved throughout the lifetime

97 (supplementary material A, part 3).

98 There is a direct, formal link between age-specific survival probabilities and lifespan

99 (Supplementary material A, part 1). For this reason, between-individual variation in survival

100 probability, which we study here, is fundamentally equivalent to between-individual variation

101 in lifespan, to which evolutionary biologists are more accustomed, but to which we do not

102 have direct access in our study populations. The between-individual heterogeneity in survival

103 probability that we quantify in this study does give rise to viability selection a.k.a. selective

104 disappearance: within the population, the proportion of frail individuals decreases with age.

105 This mechanism is, however, by construct accounted for in the estimation method (see below

106 and supplementary material A, part 3) and therefore does not bias our estimates.

107 Another major issue which we account for in our framework is that, at the population scale,

108 senescence-related declines in survival probability and between-individual heterogeneity can

109 fully or partially compensate each other (Vaupel et al. 1979; Service 2000; our supplementary

110 material A, part 4). So, ignoring senescence or relying on information theory to decide on the

111 occurrence of frailty and/or senescence can lead to downward-biased estimates of individual

112 variance (supplementary material A, part 4). We systematically accounted for senescence in

113 our estimation framework in order to remove this bias. We used the logit-linear model of

114 ageing, which is often applied to vertebrate populations (Loison et al. 1999; Bouwhuis et al.

115 2012). 


\section{CAPTURE-RECAPTURE MODELS TO ESTIMATE INDIVIDUAL HETEROGENEITY IN SURVIVAL}

117 The estimation of frailty in the wild has been the topic of intense methodological innovation

118 in recent years, all pivoting around improvements to the Cormack-Jolly-Seber capture-

119 recapture (CR) model (Pledger et al. 2003; Royle 2008; Pradel 2009; Gimenez and Choquet

120 2010). We resorted to two now well-established methods to estimate individual heterogeneity

121 of unspecified origin in survival probability: CR models with individual random effects

122 (Gimenez and Choquet 2010), and CR models with finite mixtures (Pledger et al. 2003).

123 Briefly, CR random-effect models are based on the assumption that individual heterogeneity

124 in survival follows a Gaussian distribution on the logit scale (logit-normal), being thereby

125 analogue to widely used generalized linear mixed models. CR mixture models are based on

126 the assumption that individuals can be categorized into a finite number of heterogeneity

127 classes (hidden states), i.e., the underlying distribution of frailty is approximated by a

128 "histogram-like", categorical distribution. The CR mixture models that we implemented had

129 two components: low and high survival. Both methods (i.e., mixture and random effect

130 models) allow separating process (individual) variance from sampling variance in survival

131 probability. In CR random-effect models, we used the delta method to re-scale the logit-scale

132 of between-individual variance onto the identity scale. We denoted the resulting metric $V_{R}$. In

133 CR mixture models, we used a stratified sampling formula (Eq. S2 in supplementary material

134 A). We denoted the resulting metric $V_{M}$. The two metrics $V_{R}$ and $V_{M}$ measure the same

135 quantity (individual heterogeneity in survival probability at the onset of adulthood) but use

136 different underlying models and so are expected to differ, depending on the relative fit of the

137 two models. The relative performance of the two methods (random and mixture models) was

138 assessed using model deviances and further investigated with extensive simulations

139 (supplementary material A, part 5). 
140 All CR models were fitted using program E-SURGE (Choquet et al. 2009). Detailed accounts

141 of the analytical protocols we used can be found in Péron et al. (2010) for CR mixture models

142 and Gimenez and Choquet (2010) for CR random effect models. Additional elements to

143 reproduce our CR analyses are provided in supplementary material A (part 3). In particular,

144 whether or not the study populations exhibited individual heterogeneity in capture probability

145 was assessed prior to this study in each population, and the result of that assessment was

146 carried over in our models. The statistical significance of between-individual variance was

147 assessed using likelihood ratio tests designed to accommodate the fact that the null hypothesis

148 "zero variance" is at the boundary of the parameter space (variance being always positive; see

149 Gimenez and Choquet 2010 for the technical details of the test). We also assessed whether the

150 bounded nature of survival probability itself, i.e., the fact that it must vary between zero and

151 one, acted as a constraint. Under the binomial assumption, we computed the maximum

152 variance value for mean survival probabilities varying between zero and one. We found that

153 observed between-individual variance was always much smaller than the maximum possible

154 variance under the binomial assumption. Therefore, the boundary constraint was unlikely to

155 affect the results of our interspecific comparison (supplementary material A, part 2).

\section{INTERSPECIFIC COMPARISON}

157 After obtaining estimates of between-individual variance in survival at the onset of adulthood

158 for all of our eleven study populations, we regressed species-specific variance estimates

159 against the position of the species on the slow-fast life-history continuum, in order to support

160 or infirm the canalization hypothesis. We used generation time, the weighted mean age of

161 females when they give birth, to rank species on the continuum (Gaillard et al. 2005).

162 Generation time presents the interesting property that it is directly linked to the elasticities of

163 demographic traits, i.e., the relative impact of a proportional change in trait values on the

164 population growth rate (Charlesworth 2000; Lebreton 2005). In addition, given the crucial 
165 role of allometric relationships in shaping the ranking of species along the slow-fast

166 continuum of life histories, we replicated the same regression but including the average

167 female body mass of our study populations as predictor.

168 To estimate the standard error of the regression parameters, we performed a parametric

169 bootstrap by resampling 1,000 times in the approximate multivariate normal distribution of

170 the species-specific CR models, i.e., taking the sampling variance and covariance of the

171 population-specific vital rates estimates into account (this was also used to compute standard

172 error on $V_{M}$ and $V_{R}$ estimates). Due to the relatively small number of species, we did not

173 consider phylogenetic inertia (Sæther et al. 2013). However, we incorporated a fixed class

174 effect (bird/mammal) in the above regression. These analyses were performed with R.

\section{Results}

176 As a general rule, the random-effect CR model fitted data less well than the mixture CR

177 model (deviance in supplementary material B and simulation in supplementary material A,

178 part 5). The amount of individual heterogeneity in survival at the onset of adulthood

179 decreased with increasing generation time (Fig. 1; log-log regression slope: $-2.20 \pm$ bootstrap

180 SE 0.90; correlation coefficient: $-0.22 \pm 0.16$ ) and with increasing body mass (Fig. 1; log-log

181 regression slope: $-1.06 \pm$ bootstrap SE 0.45 ; correlation coefficient: $-0.21 \pm 0.15$ ). However,

182 these relationships were mostly caused by the contrast between two short-lived, small species

183 (blue tit and white-throated dipper; Table 1) and all the other, longer-lived, heavier species.

184 Indeed, although most of the populations we studied did not exhibit any detectable individual

185 heterogeneity in survival, our findings actually show that individual heterogeneity in survival

186 at the onset of adulthood does decline from fast- to slow-living species, in line with the

187 canalization hypothesis. 


\section{Discussion}

189 Using eleven long-term time series of individual-based demographic data, we found that

190 individual heterogeneity in survival at the onset of adulthood was low and mostly

191 undetectable in long-lived species, whereas it was marked in short-lived species. In long-lived

192 species, the same variation in adult survival that we found in short-lived species would have

193 had a much greater impact on average individual fitness than in short-lived species (Pfister

194 1998). Our finding thus corroborates the hypothesis that traits whose variation has the greatest

195 potential effect on fitness are the most canalized. Reduced variation in adult survival has

196 previously been reported in large mammalian herbivores and large seabirds, but using

197 temporal, not individual, variation (Gaillard and Yoccoz 2003; Nevoux et al. 2010). Although

198 few studies have quantified individual heterogeneity in adult survival in the wild, those that

199 did so far support our findings. A bird species with a generation time of 2 years exhibited

200 detectable individual heterogeneity (Knape et al. 2011), whereas a bird species with a

201 generation time of 25 years exhibited almost none (Barbraud et al. 2013). Our result is not

202 tautological, in the sense that it is not due to the bounded space in which survival probability

203 varies between zero and one (supplementary material A, part 2), nor is it affected by the bias

204 that senescence would have generated in variance estimates if not accounted for (Service

205 2000). Rather, and even though we cannot disentangle the relative contributions of

206 environmental and genetic factors, our finding aligns with the recent analysis by Caswell

207 (2014) of the between-individual variation in lifespan. Caswell (2014) found that individual

208 heterogeneity accounted for less than $10 \%$ of the between-individual variation observed in

209 lifespan of Humans (generation time $>25$ years), whereas it accounted for between 46 and

$21083 \%$ of the individual variation in lifespan of short-lived laboratory-bred invertebrate species

211 with generation times shorter than a year. 
212 In conclusion, we provide a first systematic assessment of individual heterogeneity in adult

213 survival along the slow-fast continuum of vertebrate life histories. That only the shortest-

214 lived species with generation times shorter than 3 years exhibited detectable and substantial

215 individual heterogeneity in survival at the onset of adulthood corroborates the canalization

216 hypothesis.

\section{Acknowledgements}

218 We thank everyone involved in fieldwork and data management for the long-term monitoring

219 of marked individuals. Critical support for the long-term studies was provided by IPEV

220 program n¹09, Zone Atelier Antarctique, and TAAF; Office National de la Chasse et de la

221 Faune Sauvage; BioAdapt grant ANR-12-ADAP-0006-02-PEPS to A.C; ANR grant 08-JCJC-

222 0028-01 to O.G. This is a contribution of the GDR 3645 'Statistical Ecology'. We are most

223 grateful to Stephen Dobson for insightful comments on an earlier draft of this paper.

\section{Supplementary material}

225 Supplementary material A: Material and method complements

226 Supplementary material B: Deviances and Akaike Information Criteria.

\section{Literature cited}

228 Barbraud, C. et al. 2013. Fisheries bycatch as an inadvertent human-induced evolutionary

229 mechanism. PloS one 8:e60353.

230 Bouwhuis, S. et al. 2012. The Forms and Fitness Cost of Senescence: Age-Specific Recapture,

231 Survival, Reproduction, and Reproductive Value in a Wild Bird Population. The American

232 Naturalist 179:E15-E27.

233 Brown, J. H., and R. M. Sibly. 2006. Life-history evolution under a production constraint. 
Proceedings of the National Academy of Sciences 103:17595-17599.

235 Brown, J. H., and G. B. West. 2000. Scaling in Biology. Oxford University Press, Oxford.

236 Caswell, H. 2014. A matrix approach to the statistics of longevity in heterogeneous frailty

237 models. Demographic Research 31:553-592.

238 Caswell, H. 2001. Matrix Population Models: Construction, Analysis, and Interpretation.

239 Sinauer Associates.

240 Charlesworth, B. 2000. Fisher, Medawar, Hamilton and the evolution of aging. Genetics

$241 \quad$ 156:927-931.

242 Choquet, R. et al. 2009. Program E-SURGE: a software application for fitting multievent

243 models. Pp. 845-865 in D. L. Thomson et al., eds. Modeling demographic processes in

244 Marked Populations. Springer US, Environmental and Ecological Statistics, Springer, New

245 York.

246 Gaillard, J. M. et al. 2005. Generation time: A reliable metric to measure life-history variation

247 among mammalian populations. The American Naturalist 166:119-123.

248 Gaillard, J. M., and N. G. Yoccoz. 2003. Temporal variation in survival of mammals: A case

249 of environmental canalization? Ecology 84:3294-3306.

250 Gaillard, J.-M. et al. 2016. Axes of variation in life histories. P. in press in R. M. Kliman, ed.

251 Encyclopedia of Evolutionary Biology. Elsevier, New York.

252 Gillooly, J. F. et al. 2001. Effects of size and temperature on metabolic rate. Science

253 293:2248-2251.

254 Gimenez, O., and R. Choquet. 2010. Individual heterogeneity in studies on marked animals

255 using numerical integration: capture-recapture mixed models. Ecology 91:951-957. 
256 Harvey, P. H., and R. M. Zammuto. 1985. Patterns of mortality and age at first reproduction

257 in natural populations of mammals. Nature 315:319-320.

258 Kirkwood, T. B. C., and F. R. S. Holliday. 1979. The evolution of ageing and longevity.

259 Proceedings Of The Royal Society B-Biological Sciences 205:531-546.

260 Knape, J. et al. 2011. Individual heterogeneity and senescence in Silvereyes on Heron Island.

261 Ecology 92:813-820.

262 Koons, D. N. et al. 2009. Is life-history buffering or lability adaptive in stochastic

263 environments? Oikos 118:972-980.

264 Lebreton, J. D. 2005. Age, stages, and the role of generation time in matrix models.

265 Ecological Modelling 188:22-29.

266 Lebreton, J. D. et al. 1992. Modeling survival and testing biological hypotheses using marked

267 animals - A unified approach with case-studies. Ecological Monographs 62:67-118.

268 Loison, A. et al. 1999. Age-specific survival in five populations of ungulates: evidence of

269 senescence. Ecology 80:2539-2554.

270 Nevoux, M. et al. 2010. Bet-hedging response to environmental variability, an intraspecific

271 comparision. Ecology 91:2416-2427.

272 Péron, G. et al. 2010. Capture-recapture models with heterogeneity to study survival

273 senescence in the wild. Oikos 119:524-532.

274 Pfister, C. A. 1998. Patterns of variance in stage-structured populations: Evolutionary

275 predictions and ecological implications. Proceedings of the National Academy of Sciences

276 95:213-218.

277 Pledger, S. et al. 2003. Open capture-recapture models with heterogeneity: I. Cormack-Jolly- 
278 Seber model. Biometrics 59:786-94.

279 Pradel, R. 2009. The stakes of capture-recapture models with state uncertainty. Pp. 781-795

280 in D. L. Thomson et al., eds. Modeling Demographic Processes In Marked Populations.

281 Springer, New York.

282 Royle, J. A. 2008. Modeling individual effects in the Cormack-Jolly-Seber model: A state283 space formulation. Biometrics 64:364-370.

284 Sæther, B.-E. et al. 2013. How life history influences population dynamics in fluctuating 285 environments. American Naturalist 182:743-759.

286 Service, P. 2000. Heterogeneity in Individual Mortality Risk and Its Importance for

287 Evolutionary Studies of Senescence. The American Naturalist 156:1-13.

288 Stearns, S. C. 1976. Life-history tactics - review of ideas. Quarterly Review of Biology 51:328947.

290 Stearns, S. C. 1983. The influence of size and phylogeny on patterns of covariation among 291 life-history traits in the mammals. Oikos 41:173-187.

292 Stearns, S. C., and T. J. Kawecki. 1994. Fitness sensitivity and the canalization of life-history 293 traits. Evolution 48:1438-1450.

294 Vaupel, J. W. et al. 1979. The impact of heterogeneity in individual frailty on the dynamics of 295 mortality. Demography 16:439-54.

296 Waddington, C. 1953. Genetic Assimilation of an Acquired Character. Evolution 7:118-126. 
299

30

302

\begin{tabular}{lllllllll}
\hline & $\mathrm{T}$ (year) & $\mathrm{e}$ & $\mathrm{m}(\mathrm{kg})$ & $\mathrm{V}_{\mathrm{M}}$ & $\mathrm{V}_{\mathrm{R}}$ & $\mathrm{s}_{1}$ & $\mathrm{~s}_{2}$ & $\pi$ \\
\hline Blue tit & 2 & 0.500 & 0.01 & $\mathbf{0 . 0 3 6 1}( \pm \mathbf{0 . 0 1 8 9})$ & $0.0097( \pm 0.0064)$ & 0.29 & 0.83 & 0.31 \\
White-throated dipper & 2.5 & 0.400 & 0.06 & $\mathbf{0 . 0 3 8 5}( \pm \mathbf{0 . 0 2 3 0})$ & $\mathbf{0 . 0 3 8 2}( \pm \mathbf{0 . 0 0 4 3})$ & 0.34 & 0.84 & 0.70 \\
Roe deer (CH) & 4.5 & 0.222 & 22 & $\mathbf{9 . 6 0 E - 0 4 ~ ( \pm 8 . 6 9 E - 0 4 )}$ & $1.46 \mathrm{E}-11( \pm 3.46 \mathrm{E}-06)$ & 0.93 & 1.00 & 0.33 \\
Roe deer (3F) & 4.5 & 0.222 & 24 & $7.10 \mathrm{E}-05( \pm 2.17 \mathrm{E}-04)$ & $1.97 \mathrm{E}-10( \pm 2.96 \mathrm{E}-07)$ & 0.97 & 0.97 & 1.00 \\
Chamois & 6 & 0.167 & 31 & $\mathbf{0 . 0 0 6 4}( \pm \mathbf{0 . 0 0 5 9})$ & $1.37 \mathrm{E}-22( \pm 4.72 \mathrm{E}-20)$ & 0.88 & 0.99 & 0.10 \\
Greater Kudu (TSH) & 6 & 0.167 & 170 & $3.04 \mathrm{E}-04( \pm 2.14 \mathrm{E}-03)$ & $8.07 \mathrm{E}-08( \pm 6.55 \mathrm{E}-06)$ & 0.99 & 0.99 & 0.50 \\
Greater Kudu (PK) & 6 & 0.167 & 170 & $4.29 \mathrm{E}-04( \pm 9.23 \mathrm{E}-04)$ & $1.40 \mathrm{E}-07( \pm 4.65 \mathrm{E}-05)$ & 0.95 & 0.95 & 0.50 \\
Black-headed gull & 7 & 0.143 & 0.30 & $3.63 \mathrm{E}-04( \pm 1.55 \mathrm{E}-03)$ & $1.59 \mathrm{E}-05( \pm 2.43 \mathrm{E}-04)$ & 0.84 & 0.86 & 0.69 \\
Alpine ibex & 8 & 0.125 & 40 & $2.30 \mathrm{E}-04( \pm 8.79 \mathrm{E}-04)$ & $1.21 \mathrm{E}-04( \pm 3.85 \mathrm{E}-05)$ & 0.99 & 0.99 & 0.54 \\
Black-browed albatross & 19 & 0.053 & 4 & $0.0036( \pm 0.0073)$ & $1.47 \mathrm{E}-06( \pm 4.25 \mathrm{E}-05)$ & 0.90 & 0.95 & 0.13 \\
Snow petrel & 25 & 0.040 & 0.35 & $0.0043( \pm 0.0191)$ & $4.00 \mathrm{E}-09( \pm 2.00 \mathrm{E}-06)$ & 0.98 & 0.99 & 0.76 \\
\hline
\end{tabular}
from 1000 replicates of the parametric bootstrap between parentheses. Bold font indicates P-values $<0.05$ for the likelihood ratio test of individual high survival group, and proportion of individuals in the low survival group at first capture).

Table 1: Individual heterogeneity in survival probability of our study populations. $T$ and $m$ are the generation time and average female body mass in the study populations. $\mathrm{e}$ is the inverse of $\mathrm{T}$ and measures the impact of a given variation in recruitment rate on average individual fitness (Charlesworth 2000; Lebreton 2005). $V_{M}$ and $V_{R}$ are the estimated between-individual variances from mixture and random-effect capture-recapture models, respectively, with standard error heterogeneity. $\mathrm{s}_{1}, \mathrm{~s}_{1}$, and $\pi$ are parameter estimates from the CR mixture models (annual survival at the onset of adulthood for the low survival group, for the 


\section{Figure legends}

308 Figure 1: Between-individual variance estimate $V_{M}$ plotted against generation time (left

309 panel) and body mass (right panel). One-standard deviation confidence intervals are from a

310 parametric bootstrap with 1000 replicates. Asterisks indicate statistically significant

311 likelihood-ratio tests $(P<0.05)$. 


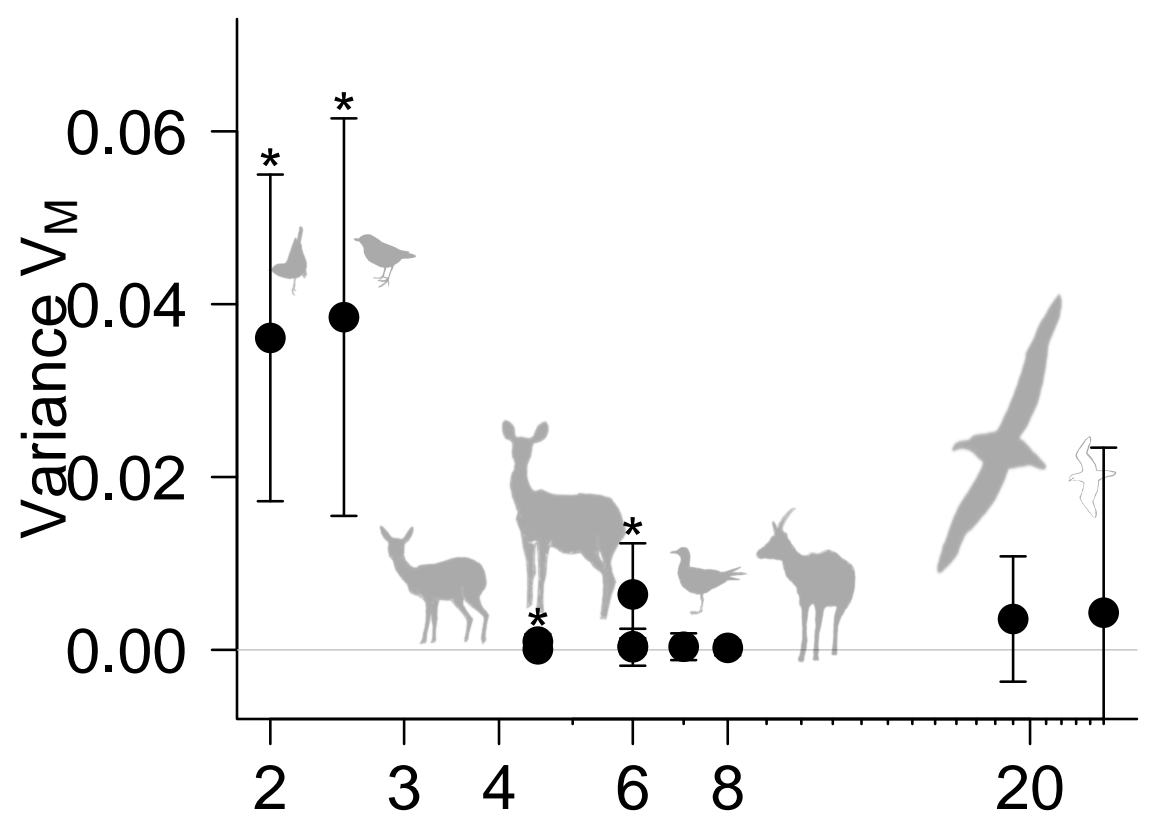

Generation time (years, log scale)

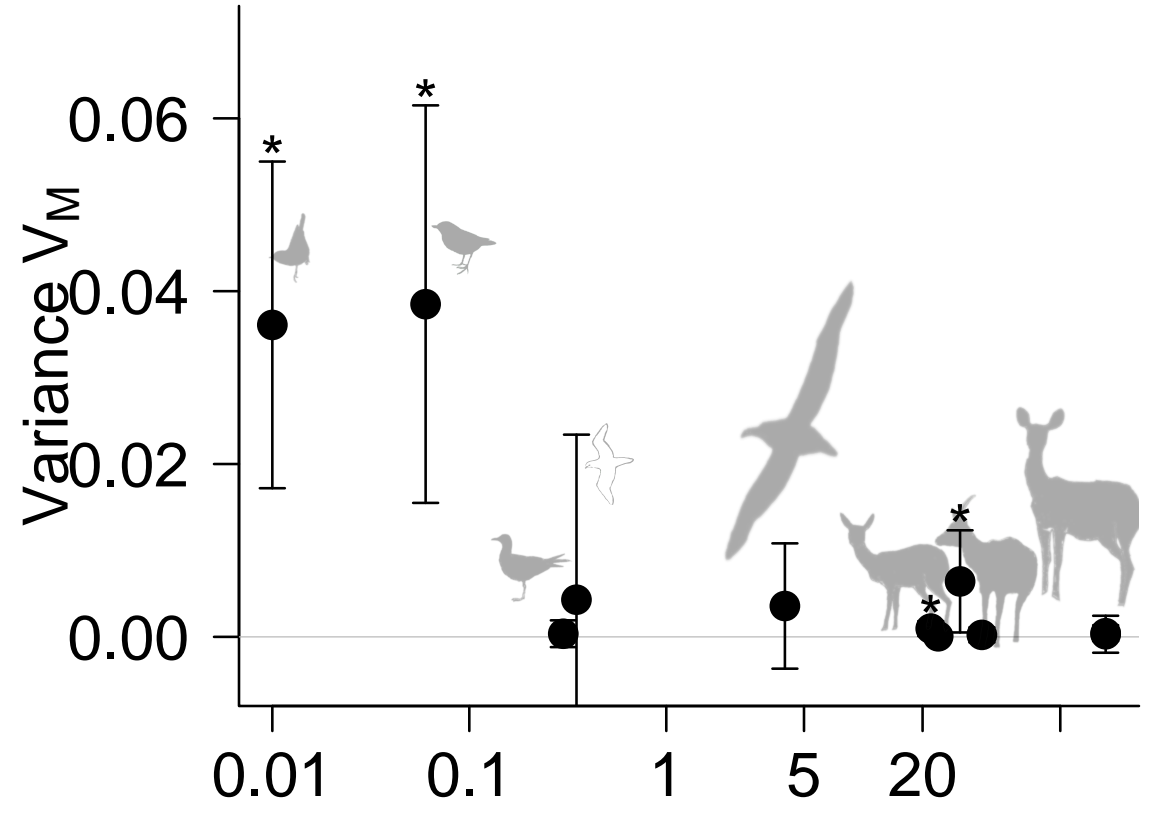

Body mass (kg, log scale) 